\title{
Boundary value problems for local and nonlocal Liouville type equations with several exponential type nonlinearities. Radial and nonradial solutions
}

\author{
Angela Slavova $^{1 *}$ and Petar Popivanov ${ }^{1}$
}

*Correspondence:

angela.slavova@gmail.com 1 Institute of Mathematics and Informatics, Bulgarian Academy of Sciences, Sofia 1113, Bulgaria

\section{Springer}

\begin{abstract}
This paper deals with boundary value problems for local and nonlocal Laplace operator in 2D with exponential nonlinearities, the so-called Liouville type equations. They include the mean field equation and other equations arising in the statistical mechanics. Existence results into an explicit form for the Dirichlet problem in the unit disc $B_{1} \subset \mathbf{R}^{2}$ and in the participation of positive parameters in the right-hand sides are proved in Theorems 2 and 3 . Theorem 2 is illustrated by several examples including an application to the differential geometry. In Theorem 4 global radial solution of the Cauchy problem with constant data at $\partial B_{1}$ and under appropriate conditions is constructed. It develops logarithmic singularities for $r=0, r=\infty$. An illustrative example to Theorem 4 in the case of two exponents is given at the end of the paper.
\end{abstract}

MSC: 35A25; 35C05; 37J35; 35Q51; 35Q55; 35Q60

Keywords: Nonlocal PDE; Liouville type elliptic equation; Dirichlet problem; Radial solution; Blaschke product; Cauchy problem

\section{Introduction}

Following the pioneering work [15] of L. Onsager (1903-1968) in the frames of the statistical mechanics of two-dimensional point vortices and the mean field equations of hydrodynamic turbulence in equilibrium, different types of nonlocal elliptic equations with exponential type nonlinearities recently have been intensively studied. In what follows we propose several papers on the subject $[2-4,6,9,16,19,25-27,30]$. Very often in investigating these boundary value problems (BVP) variational methods are applied (for example, see $[12,14])$. The advantage of this approach is that one can work in bounded smooth domains in the plane. Our aim here is to find (mainly) radial solutions to the boundary value problems for local and nonlocal PDE of Liouville type. To do this, we shall use the machinery of the classical ODE (see $[1,21-23,29]$ ) as in several cases the solutions of our PDE with constant data on the unit circumference $S_{1}=\partial B_{1}=\left\{|x|=1, x \in \mathbf{R}^{2}\right\}$ are radially symmetric $[10,24]$. The coefficients of the equations could be radial too. Topological

(c) The Author(s) 2021. This article is licensed under a Creative Commons Attribution 4.0 International License, which permits use sharing, adaptation, distribution and reproduction in any medium or format, as long as you give appropriate credit to the original author(s) and the source, provide a link to the Creative Commons licence, and indicate if changes were made. The images or other third party material in this article are included in the article's Creative Commons licence, unless indicated otherwise in a credit line to the material. If material is not included in the article's Creative Commons licence and your intended use is not permitted by statutory regulation or exceeds the permitted use, you will need to obtain permission directly from the copyright holder. To view a copy of this licence, visit http://creativecommons.org/licenses/by/4.0/. 
methods are also applied in the investigation of elliptic PDE with exponential nonlinearities, see [13].

We shall consider two nonlocal BVPs which do not possess radial solutions.

\section{Preliminary definitions and formulation of the main results}

In order to formulate the corresponding results, we propose several preliminary notes from the complex analysis. Consider the analytic function $f(z)$ in $B_{1}=\left\{z \in \mathbf{C}^{1}:|z|<1\right\}$, $f \in C^{0}\left(\bar{B}_{1}\right),\left.f\right|_{S_{1}} \neq 0$. Then $f(z)$ can vanish in finitely many points $\alpha_{1}, \ldots, \alpha_{n}$ of the unit disc $B_{1}$. Certainly, $f(z)=\prod_{j=1}^{n}\left(z-\alpha_{j}\right) h(z)$, where the analytic function $h(z)$ in $B_{1}$ is such that $h \in C^{0}\left(\bar{B}_{1}\right),\left.h\right|_{\bar{B}_{1}} \neq 0$. The zeros of $f(z)$ are counted with their multiplicities, i.e., multiple zeros are admissible too.

Proposition 1 Suppose that the analytic function $f$ in $B_{1}$ is nontrivial and $\mid f \|_{S_{1}}=1$. Then $f$ has finitely many zeros $\alpha_{j}$ (at least one) in $B_{1}$ and

$$
f(z)=e^{i \gamma} \prod_{j=1}^{n} \frac{z-\alpha_{j}}{1-\bar{\alpha}_{j} z}, \quad \gamma=\text { const } \in \mathbf{R}^{1} .
$$

Conversely, each analytic function $f$ in $B_{1+\varepsilon}, \varepsilon>0$ of the form (1) satisfies the equality $\mid f \|_{S_{1}}=1$. The function $B(z)=e^{i \gamma} \prod_{j=1}^{n} \frac{z-\alpha_{j}}{1-\bar{\alpha}_{j} z}$ is called finite Blaschke product and $\alpha_{j}^{*}=\frac{1}{\bar{\alpha}_{j}}$, $\alpha_{j} \neq 0, \alpha_{j} \in B_{1}$ is the inverse point of $\alpha_{j}$ with respect to $S_{1}$. One can see $[11,19,31]$ that $B$ verifies the inequality $\mid B^{\prime} \|_{S_{1}}>0$, and if $0<\left|\alpha_{j}\right|<1$ and $\sum_{j=1}^{n}\left(\alpha_{j}^{*}-\alpha_{j}\right) \neq 0, n \geq 4$, then $B^{\prime}$ possesses at least one zero in $B_{1}$ and at least one zero in $\mathbf{C}^{1} \backslash \bar{B}_{1}$.

Assume that

$$
\Delta u+|F(z)|^{2} e^{u}=0 \quad \text { in } B_{1},
$$

$F(z)$ being an analytic function in $\bar{B}_{1}, F \neq 0$ there. Suppose that $\Phi(z)$ is an arbitrary analytic function in $B_{1}, \Phi \in C^{0}\left(\bar{B}_{1}\right)$ and $\Phi^{\prime} \neq 0$ in $\bar{B}_{1}$. Then it is well known [19] that the general classical solution of (2) is given by the formula

$$
u=\log \frac{8\left|\Phi^{\prime}(z)\right|^{2}}{|F(z)|^{2}\left(1+|\Phi(z)|^{2}\right)^{2}} .
$$

This is the famous result of Liouville (1853) shown when $F \equiv 1$.

In the case when $\Phi^{\prime}(z)$ vanishes at finitely many points $\left\{\beta_{j}\right\}_{j=1}^{n} \subset B_{1}$ and $F(z)$ vanishes at the points $\left\{\gamma_{j}\right\}_{j=1}^{m} \subset B_{1}$, the generalized solution of (2) is defined as follows:

$$
\Delta u+|F(z)|^{2} e^{u}=4 \pi\left(\sum_{j=1}^{n} \delta\left(z-\beta_{j}\right)-\sum_{j=1}^{m} \delta\left(z-\gamma_{j}\right)\right)
$$

where $u$ is a measurable function (distribution) in the unit disc, the Dirac delta function $\delta\left(z-\beta_{j}\right)=\delta\left(x-\operatorname{Re} \beta_{j}\right) \otimes \delta\left(y-\operatorname{Im} \beta_{j}\right), z=x+i y$ and (4) is satisfied in the distribution sense. The generalized solution is given by the same formula (3). 
We shall study now the nonlocal BVP

$$
\begin{aligned}
& \Delta u+\lambda\left|B^{\prime}(z)\right|^{2} \frac{e^{u}}{\int_{B_{1}} e^{u} d x}=0 \text { in } B_{1} \backslash\left\{\alpha_{1}, \ldots, \alpha_{n}\right\}, \lambda>0 \\
& \left.u\right|_{\partial B_{1}}=0
\end{aligned}
$$

and

$$
\begin{aligned}
& \Delta u+\lambda\left|B^{\prime}(z)\right|^{2} \frac{e^{u}}{\int_{B_{1}} e^{u}\left|B^{\prime}\right|^{2} d x}=0 \quad \text { in } B_{1} \backslash\left\{\alpha_{1}, \ldots, \alpha_{n}\right\}, \lambda>0 \\
& \left.u\right|_{\partial B_{1}}=0 .
\end{aligned}
$$

To specify the formulation of our first result, put

$$
B(z)=e^{i \gamma} \prod_{j=1}^{k}\left(\frac{z-\alpha_{j}}{1-\bar{\alpha}_{j} z}\right)^{k_{j}}, \quad k_{j} \geq 1, \sum_{1}^{k} k_{j}=n .
$$

$\lambda>0$ is the spectral parameter of our nonlocal nonlinear BVP (5), (6). We use the notation $B(z)$ (coming from Blaschke) instead of $f(z)$.

Theorem 2 (a) Consider BVP (5) and suppose that at least one $k_{j}$ of $(7)$ is $\geq 2$. Then, for each value $\lambda \in(0, \infty), B V P(5)$ has a solution. Otherwise, i.e., if $k_{1}=\cdots=k_{n}=1$, there exists $\lambda_{0}>0$ and such that (5) possesses a solution only for the values of $\lambda \in\left(0, \lambda_{0}\right)$ (finite spectrum). The solution $u$ is not unique.

(b) BVP (6) has a finite spectrum $0<\lambda<\lambda_{0}$ for each $k_{j} \geq 1$. Therefore, a solution of (6) exists only for these values of $\lambda$. We shall write down the exact value of $\lambda_{0}$. Moreover, the solutions in general are neither radial nor uniquely determined. They can be written into an explicit form.

Remark 1 Assume that at least one $\alpha_{j} \in B^{1} \backslash\{0\}$. Then the corresponding solution $u$ is nonradial. $u$ is radial if $\alpha_{1}=0, k_{1} \geq 1$, and $k_{j}=0$ for $j \geq 2$.

We shall illustrate Theorem 2 by two examples of the mean field equations. Theorem 2 can be applied to the theory of minimal, non-super conformal degenerate twodimensional surfaces $M_{2}$ in $\mathbf{R}^{4}$ (see De Azevedo and Guadalupe [20, 28]). More precisely, the Gaussian curvature $K$ and the normal curvature $\chi$ satisfy the degenerate nonlinear system of PDE (see [28]):

$$
\begin{aligned}
& |B|^{2}\left(K^{2}-\chi^{2}\right)^{1 / 4} \Delta \log |\chi-K|=2(2 K-\chi), \\
& |B|^{2}\left(K^{2}-\chi^{2}\right)^{1 / 4} \Delta \log |\chi+K|=2(2 K+\chi), \\
& K^{2}>\chi^{2}, \quad K<0 .
\end{aligned}
$$

Our next result deals with two spectral parameter BVPs, namely

$$
\begin{aligned}
& \Delta u+\lambda_{1} \frac{e^{u / 2}}{\int_{B_{1}} e^{u / 2} d x}+\lambda_{2}|x|^{2} \frac{e^{u}}{\int_{B_{1}} e^{u} d x}=0 \quad \text { in } B_{1}, \lambda_{1}>0, \lambda_{2}>0 \\
& \left.u\right|_{\partial B_{1}}=0
\end{aligned}
$$




$$
\begin{aligned}
& \Delta u+\lambda_{1} \frac{e^{u / 2}}{\int_{B_{1}} e^{u / 2} d x}+\lambda_{2}|x|^{2} \frac{e^{u}}{\int_{B_{1}}|x|^{2} e^{u} d x}=0 \quad \text { in } B_{1}, \lambda_{1}>0, \lambda_{2}>0 \\
& \left.u\right|_{\partial B_{1}}=0 .
\end{aligned}
$$

Theorem 3 Consider BVP (9). It has a radial solution $u(r, \mu), \mu=\left(\mu_{1}, \mu_{2}\right), \mu_{1}>0, \mu_{2}>0$ of the form

$$
u=\log \frac{B_{0}^{2}(\mu)}{\left[a_{0}+\frac{\mu_{1} B_{0}}{8} r^{2}+\frac{B_{0}^{2} r^{4}}{32 a_{0}}\left(\frac{\mu_{1}^{2}}{8}+\mu_{2}\right)\right]^{2}},
$$

where $a_{0}>0$ is a parameter, $B_{0}(\mu)>0$ is a function of $\left(\mu_{1}, \mu_{2}\right)$ and $\mu \in \tilde{\Delta}=\left\{0<\mu_{1} \leq 8,0<\right.$ $\left.\mu_{1} \leq 4, \mu_{1}+\frac{\mu_{2}}{2} \leq \frac{1}{4}\right\}$ satisfy the transcendental system

$$
\begin{aligned}
& 0<\lambda_{1}=F_{1}\left(\mu_{1}, \mu_{2}\right), \\
& 0<\lambda_{2}=F_{2}\left(\mu_{1}, \mu_{2}\right) .
\end{aligned}
$$

The functions $F_{1}, F_{2}$ are written explicitly in what follows.

The solution $u$ is obtained by putting the inverse functions $\mu_{1}=G_{1}\left(\lambda_{1}, \lambda_{2}\right), \mu_{2}=$ $G_{2}\left(\lambda_{1}, \lambda_{2}\right)$ of (12) into (11). The above $2 \times 2$ mapping is smoothly invertible for almost all points $\left(\lambda_{1}, \lambda_{2}\right)$.

The study of (10) is left to the reader.

We shall find the solutions of (8) vanishing at $\alpha_{j}$, as $B=B(z)$ is the Blaschke finite product. Our next step is to study the non-correct Cauchy problem in the unit disc for the Laplace operator equipped with a linear combination of exponential nonlinearities with radial coefficients. The notion of correct Cauchy problem contains the existence of a unique solution and its continuous dependence on the initial data. The Cauchy problem for Laplace operator with initial data for $t=0$ is non-correct.

Theorem 4 (a) Consider the local non-correct Cauchy problem

$$
\Delta u+\sum_{j=1}^{n} \mu_{j}|x|^{\rho_{j}} e^{\kappa_{j} u}=0 \quad \text { in } B_{1},
$$

$\mu_{j}>0, \kappa_{1}>\kappa_{2}>\cdots>0$ and either $\rho_{j^{\prime}} \geq 1$ for some $j^{\prime}$ or $\rho_{j^{\prime \prime}}=0,\left.u\right|_{\partial B_{1}}=u_{0}=$ const, $\left.\frac{\partial u}{\partial n}\right|_{\partial B_{1}}=$ $u_{1}=$ const.

\section{Under the condition}

(i) $\frac{\rho_{1}+2}{\kappa_{1}}=\frac{\rho_{2}+2}{\kappa_{2}}=\cdots=\frac{\rho_{n}+2}{\kappa_{n}}=-A>0$,

Equation (13) possesses for $1 \geq r>0$ a unique smooth radial solution $u(r, \mu), \mu=$ $\left(\mu_{1}, \ldots, \mu_{n}\right)$ which can be prolonged globally for $r>1$. The function $u$ has logarithmic singularities at $r=0$ and $r=\infty$ of the following types: $u \sim \log r^{A-\sqrt{2 C}}$ for $r \rightarrow \infty$, where $C(\mu)$ depends also on $\left(u_{0}, u_{1}\right)$ and is written explicitly, $u \sim \log r^{A+\sqrt{2 C}}, r \rightarrow 0$. 
(b) The nonlocal BVP

$$
\begin{aligned}
& \Delta u+\sum_{j=1}^{n} \lambda_{j}|x|^{\rho_{j}} \frac{e^{\kappa_{j} u}}{\int_{B_{1}} e^{\kappa_{j} u} d x}=0 \quad \text { in } B_{1}, \lambda_{j}>0 \\
& \left.u\right|_{\partial B_{1}}=u_{0},\left.\frac{\partial u}{\partial n}\right|_{\partial B_{1}}=u_{1}
\end{aligned}
$$

under the requirements $(i)$ and $\kappa_{j} \sqrt{2 C(\mu)}>\rho_{j}, j=1, \ldots, n$, has the solution $u(r, \mu)$ constructed in $(a)$, where $\lambda=\left(\lambda_{1}, \ldots, \lambda_{n}\right)$ satisfies the transcendental system

$$
0<\lambda_{j}=\mu_{j} \int_{B_{1}} e^{\kappa_{j} u} d x \equiv F_{j}(\mu), \quad 1 \leq j \leq n,
$$

the corresponding integrals in $F_{j}(\mu)$ are convergent, and the symbols $\mu_{j}=\mu_{j}(\lambda), 1 \leq j \leq n$ stand for the solutions of system (15).

Then $u=u(r, \mu(\lambda))$ verifies (14).

As it is evident, the solvability of (15) is rather complicated, uneffectively. As is concerns the solution $u$ of (13), it can be written as $u=w+A \log r, \log r=\frac{1}{\sqrt{2}} \int_{w_{0}}^{w(r)} \frac{d z}{\sqrt{C-\sum_{1}^{n} B_{j} e^{k_{j} z}}}$, where $B_{j}=\frac{\mu_{j}}{\kappa_{j}}>0$ and $2 C=w_{1}^{2}+2 \sum_{1}^{n} B_{j} e^{\kappa_{j} w_{0}}, w(0)=u_{0}, w^{\prime}(0)=u_{1}-A$.

We shall illustrate Theorem 4 (a), case $\kappa_{1}=1, \kappa_{2}=1 / 2, \rho_{1}=2, \rho_{2}=0 \Rightarrow A=-4$, by a solution $u$ that is expressed as a logarithm of the radial function of $r^{1 / 2 \sqrt{2 C}}$. In the case $\kappa_{1}=1, \kappa_{2}=2, \kappa_{3}=3, \rho_{1}=1, \rho_{2}=4, \rho_{3}=7 \Rightarrow A=-3$, the solution $w(r, \mu)$ is expressed by Legendre's elliptic functions of first and third kind [5].

The paper is organized as follows. In Sect. 3 Theorem 2 is proved and radial and nonradial solutions are found with applications to geometry. In Sect. 4 Theorem 3 is shown. In Sect. 5 Theorem 4 is proved and an illustrative example is proposed. The solution is given explicitly as a rational function of two exponents.

\section{Proof of Theorem $\mathbf{2}$ and an application to the differential geometry}

The main idea of the proof is to localize $\int_{B_{1}} e^{u} d x$, respectively $\int_{B_{1}}\left|B^{\prime}(z)\right|^{2} e^{u} d x$, near the zeroes $\alpha_{j}$ of $B(z)$. Thus, take $\Phi=C B(z), C=$ const $>0$ in (3), i.e., $F=\Phi^{\prime}(z) \Rightarrow u=$ $\log \frac{8 C^{2}}{\lambda\left(1+C^{2}|B|^{2}\right)^{2}}$. $C$ will be determined further on, and it takes two values: $C_{+}(\lambda), C_{-}(\lambda)$. So $\left.u\right|_{\partial B_{1}}=0 \Longleftrightarrow 1=\frac{2 \sqrt{2} C}{\sqrt{\lambda}\left(1+C^{2}\right)}$ as $\mid B(z) \|_{S_{1}}=1$. Therefore, $C_{ \pm}(\lambda)=\frac{\sqrt{2}\left(1 \pm \sqrt{1-\frac{\lambda}{2}}\right)}{\sqrt{\lambda}}, 0<\lambda \leq 2 \Rightarrow$ $C_{+}(\lambda)>C_{-}(\lambda)$ for $0<\lambda<2, C_{ \pm}(2)=1$. Therefore, $C_{+}(\lambda) \sim 2 \sqrt{\frac{2}{\lambda}}, \lambda \rightarrow 0, C_{-}(\lambda) \sim \frac{\sqrt{2}}{4} \sqrt{\lambda}$, $\lambda \rightarrow 0$, i.e., $C_{-}(\lambda)$ is bounded in $(0,2]$.

In the case (5) we denote $\frac{\lambda}{\mu}=\int_{B_{1}} e^{u} d x$, and in the case (6) we put $\frac{\lambda}{\mu}=\int_{B_{1}}\left|B^{\prime}(z)\right|^{2} e^{u} d x$, $\mu>0$. So (5), (6) take the same form but with different $\mu>0$ :

$$
\begin{aligned}
& \Delta u+\mu\left|B^{\prime}(z)\right|^{2} e^{u}=0 \quad \text { in } B_{1}, \\
& \left.u\right|_{S_{1}}=0, \\
& \Delta u+\mu\left|B^{\prime}(z)\right|^{2} e^{u}=0 \quad \text { in } B_{1}, \\
& \left.u\right|_{S_{1}}=0 .
\end{aligned}
$$


The solutions of (16), (17) are given by the formula

$$
u=\log \frac{8 C_{ \pm}^{2}(\mu)}{\mu\left(1+C_{ \pm}^{2}(\mu)|B|^{2}\right)^{2}}
$$

and $C_{ \pm}(\mu)=\sqrt{\frac{2}{\mu}}(1 \pm \sqrt{1-\mu / 2}), 0<\mu \leq 2, C_{+}(\mu)>C_{-}(\mu)$ for $0<\mu<2, C_{ \pm}(2)=1$. Moreover, $C_{-}^{\prime}(\mu)>0$ for $0<\mu<2$, while $C_{+}^{\prime}(\mu)<0$, i.e., $0<\mu_{1}<\mu_{2}<2 \Rightarrow C_{-}\left(\mu_{1}\right) \neq C_{-}\left(\mu_{2}\right)$ and $C_{+}\left(\mu_{1}\right) \neq C_{+}\left(\mu_{2}\right)$.

To solve (5), (6) we must solve the transcendental equations

$$
\begin{aligned}
& \frac{\lambda}{\mu}=\int_{B_{1}} e^{u} d x=\frac{8 C^{2}}{\mu} \int_{B_{1}} \frac{d x}{\left(1+C^{2}|B|^{2}\right)^{2}}, \\
& \frac{\lambda}{\mu}=\int_{B_{1}} \frac{\left|B^{\prime}\right|^{2} d x}{\left(1+C^{2}|B|^{2}\right)^{2}} \cdot \frac{8 C^{2}}{\mu},
\end{aligned}
$$

i.e.,

$$
\begin{aligned}
& \frac{\lambda}{8}=C_{ \pm}^{2}(\mu) \int_{B_{1}} \frac{d x}{\left(1+C^{2}|B|^{2}\right)^{2}}=G_{1, \pm}(\mu), \quad 0<\mu \leq 2 \\
& \frac{\lambda}{8}=C_{ \pm}^{2}(\mu) \int_{B_{1}} \frac{\left|B^{\prime}\right|^{2} d x}{\left(1+C^{2}|B|^{2}\right)^{2}}=G_{2, \pm}(\mu), \quad 0<\mu \leq 2 .
\end{aligned}
$$

As we mentioned,

$$
\int_{B_{1}}(\ldots)=\sum_{j=1}^{m} \int_{B_{\varepsilon}\left(\alpha_{j}\right)}(\ldots)+\int_{B_{1} \backslash \cup B_{\varepsilon}\left(\alpha_{j}\right)}
$$

where $B_{\varepsilon}\left(\alpha_{j}\right)=\left\{z:\left|z-\alpha_{j}\right| \leq \varepsilon\right\}, 0<\varepsilon \ll 1$.

As in $B_{1} \backslash \cup B_{\varepsilon}\left(\alpha_{j}\right)=D$, the function $|B|$ is bounded, i.e., $0<C_{0}<|B(z)| \leq C_{1}$ and $\left|B^{\prime}\right|$ is bounded, we conclude that $\int_{D}(\ldots) \rightarrow 0$ for $\mu \rightarrow 0$. In fact $C_{-}^{2}(\mu) \sim \frac{1}{8} \mu, \mu \rightarrow 0$. In the case $C_{+}^{2}(\mu) \sim \frac{8}{\mu}, \mu \rightarrow 0$ we have again that $\int_{D}(\ldots) \rightarrow 0$ for $\mu \rightarrow 0$. As it concerns $\int_{B_{\varepsilon}\left(\alpha_{j}\right)}(\ldots)$, after a translation it is reduced to the estimation of the integrals

$$
I_{j}=C_{ \pm}^{2}(\mu) \int_{B_{\varepsilon}(0)} \frac{d x}{\left(1+C_{ \pm}^{2}|z|^{2 k_{j}}\left|h_{j}(z)\right|^{2}\right)^{2}}, \quad h_{j}(0) \neq 0
$$

respectively

$$
I I_{j}=C_{ \pm}^{2}(\mu) k_{j}^{2} \int_{B_{\varepsilon}(0)} \frac{|z|^{2 k_{j}-2}\left|g_{j}(z)\right|^{2} d x}{\left(1+C_{ \pm}^{2}|z|^{2 k_{j}}\left|h_{j}(z)\right|^{2}\right)^{2}}
$$

where $z=x_{1}+i x_{2},|z|^{2}=x_{1}^{2}+x_{2}^{2}, g_{j}(0) \neq 0, g_{j}, h_{j}$ being analytic. De facto $g_{j}(z)=g_{j}\left(\alpha_{j}+r e^{i \varphi}\right)$ etc. We shall study only the case $I I_{j}$ as it seems to be more complicated. The case $I_{j}$ is considered in a similar way. Thus,

$$
I I_{j \pm}(\mu)=C_{ \pm}^{2}(\mu) k_{j}^{2} \int_{0}^{\varepsilon} \int_{0}^{2 \pi} \frac{r^{2 k_{j}-1}\left|g_{j}\left(r e^{i \varphi}\right)\right|^{2} d r d \varphi}{\left(1+C_{ \pm}^{2}(\mu) r^{2 k_{j}}\left|h_{j}\left(r e^{i \varphi}\right)\right|^{2}\right)^{2}}
$$


One can easily see that $G_{1-}(+0)=G_{2-}(+0)=0, G_{1 \pm}(2)=\int_{B_{1}} \frac{d x}{\left(1+|B|^{2}\right)^{2}}, G_{2 \pm}(2)=\int_{B_{1}} \frac{\left|B^{\prime}\right|^{2} d x}{\left(1+|B|^{2}\right)^{2}}$. Consequently, we must find $G_{2+}(0)$.

The change $r^{k_{j}} C_{+}=z$ in the integral $I I_{+}(\mu)$ leads to

$$
I I_{j+}(\mu)=k_{j} \int_{0}^{\varepsilon^{k_{j}} C_{+}} \int_{0}^{2 \pi} \frac{z\left|g_{j}\left(\frac{z^{\frac{1}{k_{j}}}}{C_{+}^{1 / k_{j}}} e^{i \varphi}\right)\right|^{2} d z d \varphi}{\left(1+z^{2}\left|h_{j}\left(z^{\frac{1}{k_{j}}} C_{+}^{1 / k_{j}} e^{i \varphi}\right)\right|^{2}\right)^{2}}
$$

$\rightarrow_{\mu \rightarrow 0} k_{j} \int_{0}^{\infty} \int_{0}^{2 \pi} \frac{z d z d \varphi\left|g_{j}(0)\right|^{2}}{\left(1+z^{2}\left|h_{j}(0)\right|^{2}\right)^{2}}=\pi \frac{\left|g_{j}(0)\right|^{2}}{\left|h_{j}(0)\right|^{2}} k_{j}$ according to the Lebesgue dominated convergence theorem.

Conclusion $G_{2+}(0)=\pi \sum_{j=1}^{m} k_{j} \frac{\left|g_{j}\left(\alpha_{j}\right)\right|^{2}}{\left|h_{j}\left(\alpha_{j}\right)\right|^{2}}$.

Therefore, $\mu \in(0,2) \Rightarrow G_{2-}(0)=0, G_{2-}(2)=G_{2+}(2)=\int_{B_{1}} \frac{\left|B^{\prime}\right|^{2} d x}{\left(1+|B|^{2}\right)^{2}}, G_{2+}(0)=\pi \sum_{1}^{m} k_{j} \times$ $\frac{\left|g_{j}\left(\alpha_{j}\right)\right|^{2}}{\left|h_{j}\left(\alpha_{j}\right)\right|^{2}}$.

For BVP (6), the spectral parameter $\lambda \in\left(0,8 G_{2 \pm}(2)\right] \cup\left(8 G_{ \pm}(2), 8 G_{2+}(0)\right)$.

In case (5),

$$
\lambda \in\left(0,8 G_{1 \pm}(2)\right] \cup\left(8 G_{1 \pm}(2),\left\{\begin{array}{l}
\infty \quad \text { if at least one } k_{j} \geq 2 \\
8 \pi \sum_{1}^{n} \frac{1}{\left|h_{j}\left(\alpha_{j}\right)\right|^{2}}, k_{1}=\cdots=k_{n}=1
\end{array}\right\}\right)
$$

$\left(G_{1 \pm}(2)=\int_{B_{1}} \frac{d x}{\left(1+|B|^{2}\right)^{2}}\right)$.

Theorem 2 is proved.

Solving the transcendental Eqs. (21), (22), finding $\mu=\mu(\lambda) \in(0,2]$, and inserting it in

$$
u_{ \pm}=\log \frac{8 C_{ \pm}^{2}(\mu)}{\mu\left(1+C_{ \pm}^{2}(\mu)|B(z)|^{2}\right)^{2}}
$$

we obtain the solutions of our nonlocal nonlinear BVP (5), (6). The nonuniqueness of the solutions of (5), (6) was established in the considerations for $C_{ \pm}(\mu)$ after formula (18).

We give several examples.

Example 1 Consider the nonlocal BVP

$$
\begin{aligned}
& \Delta u+\lambda \frac{e^{u}}{\int_{B_{1}} e^{u} d x}|x|^{2}=0 \quad \text { in } B_{1}, \lambda>0 \\
& \left.u\right|_{\partial B_{1}}=0 .
\end{aligned}
$$

Then the spectral parameter $\lambda \in(0, \infty)=\left(0,8 \pi+4 \pi^{2}\right] \cup\left(8 \pi+4 \pi^{2}, \infty\right)$. The mapping

$$
\lambda=64 \pi C \int_{0}^{1} \frac{r d r}{\left(C+r^{4}\right)^{2}}
$$

where $C=C_{ \pm}(\mu)=\frac{16-\mu \pm 16 \sqrt{1-\mu / 8}}{\mu}, \mu \in(0,8)$, is invertible in both subintervals $\left(0,8 \pi+4 \pi^{2}\right]$ and $\left(8 \pi+4 \pi^{2},+\infty\right), \mu=\mu(\lambda)$ and the solutions are $u_{ \pm}=\log \frac{32 C_{ \pm}(\mu)}{\mu\left(C_{ \pm}+r^{4}\right)^{2}}$. 
Example 2

$$
\begin{aligned}
& \Delta u+\lambda \frac{|x|^{2} e^{u}}{\int_{B_{1}}|x|^{2} e^{u} d x}=0 \quad \text { in } B_{1}, \lambda>0 \\
& \left.u\right|_{\partial B_{1}}=0 .
\end{aligned}
$$

Then $\lambda \in(0,16 \pi)=(0,8 \pi] \cup(8 \pi, 16 \pi)$, the mapping $\lambda=\frac{8 \pi}{1 \pm \sqrt{1-\mu / 8}}$ is invertible in both subintervals, $0<\mu \leq 8$ and $u_{ \pm}=\log \frac{32 C_{ \pm}}{\mu\left(1+C_{ \pm} r^{4}\right)}$. More precisely, $\mu=\frac{\lambda}{\pi^{2}}\left(2 \pi-\frac{\lambda}{8}\right)$.

Example 3 To find solutions of system (8), we put $0>K-\chi=-e^{u}, 0>K+\chi=-e^{\nu} \Rightarrow K=$ $-\frac{e^{u}+e^{v}}{2}, \chi=\frac{e^{u}-e^{v}}{2}$. Thus, (8) takes the form

$$
\begin{aligned}
& |B| e^{\frac{u+v}{4}} \Delta u=-\left(3 e^{u}+e^{v}\right), \\
& |B| e^{\frac{u+v}{4}} \Delta v=-\left(e^{u}+3 e^{v}\right) .
\end{aligned}
$$

Put $p=\frac{3 u-v}{4}, q=\frac{3 v-u}{4}$ and rewrite (28) as

$$
\begin{aligned}
& |B| \Delta u=-\left(3 e^{p}+e^{q}\right), \\
& |B| \Delta v=-\left(e^{p}+3 e^{q}\right) .
\end{aligned}
$$

Thus,

$$
\begin{aligned}
& |B| \Delta p=|B| \frac{3 \Delta u-\Delta v}{4}=-2 e^{p}, \\
& |B| \Delta q=-2 e^{q} .
\end{aligned}
$$

This way system (29) reduces to two scalar equations:

$$
\begin{aligned}
& |B| \Delta p+2 e^{p}=0, \\
& |B| \Delta q+2 e^{q}=0 .
\end{aligned}
$$

Put the extra condition $\left.p\right|_{\partial B_{1}}=0,\left.q\right|_{\partial B_{1}}=0$. Then with $\mu=2, \Phi=C_{1} z, \Phi=C_{2} z, p=$ $\log \frac{4 C_{1}^{2}|B|}{\left(1+C_{1}^{2}|z|^{2}\right)^{2}}, q=\log \frac{4 C_{2}^{2}|B|}{\left(1+C_{2}|z|^{2}\right)^{2}}, C_{1}>0, C_{2}>0$, i.e., $C_{1}=1, C_{2}=1$. Consequently, $u=\frac{q+3 p}{2}$, $v=\frac{p+3 q}{2}$ and $u=\log \frac{16|B|^{2}}{\left(1+|z|^{2}\right)^{4}}, v=\log \frac{16|B|^{2}}{\left(1+|z|^{2}\right)^{2}}$.

This way we obtain that $K=-\frac{16|B|^{2}}{\left(1+|z|^{2}\right)^{4}}, \chi=0$. Certainly, avoiding the condition $\left.p\right|_{\partial B_{1}}=$ $\left.q\right|_{\partial B_{1}}=0$, we shall obtain a two-parametric family of solutions of our system:

$$
\begin{gathered}
K=\frac{-8|B|^{2} C_{1} C_{2}}{\left(1+C_{1}^{2}|z|^{2}\right)\left(1+C_{2}^{2}|z|^{2}\right)}\left(\frac{C_{1}^{2}}{\left(1+C_{1}^{2}|z|^{2}\right)^{2}}+\frac{C_{2}^{2}}{\left(1+C_{2}^{2}|z|^{2}\right)^{2}}\right), \\
\chi=\frac{8|B|^{2} C_{1} C_{2}}{\left(1+C_{1}^{2}|z|^{2}\right)\left(1+C_{2}^{2}|z|^{2}\right)}\left(\frac{C_{1}^{2}}{\left(1+C_{1}^{2}|z|^{2}\right)^{2}}-\frac{C_{2}^{2}}{\left(1+C_{2}^{2}|z|^{2}\right)^{2}}\right), \\
C_{1}>0, C_{2}>0 ; B\left(\alpha_{j}\right)=0 \Rightarrow K\left(\alpha_{j}\right)=0, \chi\left(\alpha_{j}\right)=0 .
\end{gathered}
$$




\section{Proof Theorem 3}

To begin with, we shall find a radial solution of the Liouville equation containing two exponential nonlinearities (see [17]):

$$
\Delta u+\lambda_{1} e^{u / 2}+\lambda_{2}|x|^{2} e^{u}=0 \quad \text { in } B_{1}, \lambda_{1}>0, \lambda_{2}>0 .
$$

We shall look for a solution of (32) having the form $u=\log \left(B_{0}^{2} \varphi^{2 A}\right) \Rightarrow e^{u}=B_{0}^{2} \varphi^{2 A}, e^{u / 2}=$ $B_{0} \varphi^{A}, B_{0}>0$.

Evidently,

$$
\varphi \varphi^{\prime \prime}-\left(\varphi^{\prime}\right)^{2}+\frac{\varphi \varphi^{\prime}}{r}=-\frac{1}{2 A}\left(\lambda_{1} B_{0} \varphi^{A+2}+\lambda_{2} B_{0}^{2} \varphi^{2 A+2} r^{2}\right), \quad 0 \leq r<1 .
$$

We take $A=-1 \Rightarrow-\frac{1}{2 A}=\frac{1}{2} \Rightarrow \varphi^{A+2}=\varphi, 2 A+2=0$.

Assume that $\varphi(r)=a_{0}+a_{2} r^{2}+a_{4} r^{4}, a_{i}$ being unknown constants, $a_{0}>0$ and such that $\varphi(r)>0$ for $r \geq 0$. Thus,

$$
\varphi\left(\varphi^{\prime \prime}+\frac{\varphi^{\prime}}{r}-\frac{\lambda_{1} B_{0}}{2}\right)-\left(\varphi^{\prime}\right)^{2}=\frac{\lambda_{2} B_{0}^{2}}{2} r^{2} .
$$

Inserting the expression for $\varphi(r)$ into (34) and equalizing the coefficients in front of the same powers of $r$ in the left- and right-hand sides of (34), we get

$$
a_{2}=\frac{\lambda_{1} B_{0}}{8}, a_{4}=\frac{4 a_{2}^{2}+\frac{\lambda_{2}}{2} B_{0}^{2}}{16 a_{0}}=\frac{B_{0}^{2}}{32 a_{0}}\left(\frac{\lambda_{1}^{2}}{8}+\lambda_{2}\right),
$$

where $a_{0}>0$ and $B_{0}$ are parameters.

Then the radial solution $u$ of (32) can be written as a two-parameter family of smooth functions:

$$
u=\log \frac{B_{0}^{2}}{\left(a_{0}+\frac{\lambda_{1} B_{0}}{8} r^{2}+\frac{B_{0}^{2} r^{4}}{32 a_{0}}\left(\frac{\lambda_{1}^{2}}{8}+\lambda_{2}\right)\right)^{2}} .
$$

We require $a_{0}+\frac{\lambda_{1} B_{0}}{8} r^{2}+\frac{B_{0}^{2} r^{4}}{32 a_{0}}\left(\frac{\lambda_{1}^{2}}{8}+\lambda_{2}\right)>0$ for each $r \geq 0$. Having in mind that the discriminant of that polynomial $\Delta_{1}=-\frac{1}{8} B_{0}^{2} \lambda_{2}<0$, we conclude that the latter condition holds. To solve the Dirichlet problem $\left.u\right|_{\partial B_{1}}=0$ for (32), we must have

$$
B_{0}=a_{0}+\frac{\lambda_{1} B_{0}}{8}+\frac{B_{0}^{2}}{32 a_{0}}\left(\frac{\lambda_{1}^{2}}{8}+\lambda_{2}\right),
$$

i.e., we obtain a quadratic equation with respect to $B_{0}>0$

$$
\frac{B_{0}^{2}\left(\frac{\lambda_{1}^{2}}{8}+\lambda_{2}\right)}{32 a_{0}}+B_{0}\left(\frac{\lambda_{1}}{8}-1\right)+a_{0}=0 .
$$

We suppose at first that the discriminant of (38) $\Delta_{2}=\left(\frac{\lambda_{1}}{8}-1\right)^{2}-\frac{1}{8}\left(\frac{\lambda_{1}^{2}}{8}+\lambda_{2}\right) \geq 0$, i.e.,

$$
1 \geq 1 / 4\left(\lambda_{1}+\frac{\lambda_{2}}{2}\right)
$$


In this case (38) has two real roots of the same sign. That is why we assume that

$$
0<\lambda_{1} \leq 8
$$

According to (39), $0<\lambda_{1}<4,4 \geq \lambda_{1}+\frac{\lambda_{2}}{2}, 0<\lambda_{2}<8$.

So the solution $B_{0}$ of (38) is

$$
B_{01,2}\left(\lambda_{1}, \lambda_{2}\right)=16 a_{0} \frac{1-\frac{\lambda_{1}}{8} \pm \sqrt{1-\frac{1}{4}\left(\lambda_{1}+\frac{\lambda_{2}}{2}\right)}}{\frac{\lambda_{1}^{2}}{8}+\lambda_{2}}, \quad a_{0}>0 .
$$

Consequently, (36) with $B_{01,2}$ expressed by (41) gives us a radial solution of the Dirichlet problem $\left.u\right|_{\partial B_{1}}=0$ for (32).

This is the Dirichlet BVP for nonlocal Liouville equation with two exponential nonlinearities (deterministic problem):

$$
\begin{aligned}
& \Delta u+\lambda_{1} \frac{e^{u / 2}}{\int_{B_{1}} e^{u / 2} d x}+\lambda_{2}|x|^{2} \frac{e^{u}}{\int_{B_{1}} e^{u} d x}=0 \quad \text { in } B_{1}, \lambda_{1}>0, \lambda_{2}>0, \\
& \left.u\right|_{\partial B_{1}}=0 .
\end{aligned}
$$

Denote $0<\mu_{1}=\frac{\lambda_{1}}{\int_{B_{1}} e^{u / 2} d x}, 0<\mu_{2}=\frac{\lambda_{2}}{\int_{B_{1}} e^{u} d x}$, i.e.,

$$
\begin{aligned}
& \Delta u+\mu_{1} e^{u / 2}+\mu_{2}|x|^{2} e^{u}=0 \quad \text { in } B_{1} \\
& \left.u\right|_{\partial B_{1}}=0 .
\end{aligned}
$$

We studied before this local Dirichlet BVP (32). The only difference between (32) and (43) is that we have to write $\mu_{1}, \mu_{2}$ instead of $\lambda_{1}, \lambda_{2}$ in formula (36) for the radial solution of (43). Our last step is to compute the integrals

$$
I_{1}=\int_{B_{1}} e^{u / 2} d x>0, \quad I_{2}=\int_{B_{1}} e^{u} d x
$$

by using appropriate formulas from [8]. Thus,

$$
\frac{\lambda_{1}}{\mu_{1}}=I_{1}=2 \pi B_{0} \int_{0}^{1} \frac{r d r}{\left(a_{0}+a_{2} r^{2}+a_{4} r^{4}\right)} .
$$

The change $y=r^{2}, a_{2}^{2}-4 a_{0} a_{4}<0, a_{0}>0, a_{2}>0, a_{4}>0$ leads to

$$
I_{1}=\frac{\pi B_{0}}{\sqrt{K}}\left[\operatorname{arctg} \frac{a_{4}}{\sqrt{K}}\left(1+\frac{a_{2}}{2 a_{4}}\right)-\operatorname{arctg} \frac{a_{2}}{2 \sqrt{K}}\right],
$$

where $a_{2}=\frac{\mu_{1} B_{0}}{8}, a_{4}=\frac{B^{2}}{32 a_{0}}\left(\frac{\mu_{1}^{2}}{8}+\mu_{2}\right), B_{01,2}\left(\mu_{1}, \mu_{2}\right)=\frac{1-\frac{\mu_{1}}{8} \pm \sqrt{1-\frac{1}{4}\left(\mu_{1}+\frac{\mu_{2}}{2}\right)}}{\frac{\mu_{1}^{2}}{8}+\mu_{2}} 16 a_{0}, K=a_{0} a_{4}-\frac{a_{2}^{2}}{4}=$ $\frac{B_{0}^{2} \mu_{2}}{32}$. 
This way we obtain that

$$
\begin{aligned}
\lambda_{1} & =F_{1}\left(\mu_{1}, \mu_{2}\right) \\
& =\frac{8 \pi \mu_{1}}{\sqrt{2 \mu_{2}}}\left[\operatorname{arctg}\left(\frac{B_{0}\left(\frac{\mu_{1}^{2}}{8}+\mu_{2}\right)}{4 a_{0} \sqrt{2 \mu_{2}}}\left(1+\frac{2 \mu_{1} a_{0}}{B_{0}\left(\frac{\mu_{1}^{2}}{8}+\mu_{2}\right)}\right)\right)-\operatorname{arctg} \frac{\mu_{1}}{2 \sqrt{2 \mu_{2}}}\right]
\end{aligned}
$$

in the open triangle $R=\left\{\left(\mu_{1}, \mu_{2}\right): 0<\mu_{1}<4,0<\mu_{2}<8,4>\mu_{1}+\frac{\mu_{2}}{2}\right\}$.

Compute now the integral $I_{2}[8]$ :

$$
I_{2}(r)=\pi B_{0}^{2} \int \frac{d r^{2}}{\left(a_{0}+a_{2} r^{2}+a_{4} r^{4}\right)^{2}} .
$$

One can easily see that

$$
\begin{aligned}
I_{2}= & I_{2}(1)-I_{2}(0) \\
= & \frac{16 \pi}{\mu_{2}}\left[\frac{1+\frac{2 \mu_{1} a_{0}}{B_{0}\left(\frac{\mu_{1}^{2}}{8}+\mu_{2}\right)}}{\left(1+\frac{2 \mu_{1} a_{0}}{B_{0}\left(\frac{\mu_{1}^{2}}{8}+\mu_{2}\right)}\right)+\frac{32 \mu_{2} a_{0}^{2}}{B_{0}^{2}\left(\frac{\mu_{1}^{2}}{8}+\mu_{2}\right)}}\right. \\
& +\frac{B_{0}\left(\frac{\mu_{1}^{2}}{8}+\mu_{2}\right)}{4 a_{0} \sqrt{2 \mu_{2}}} \operatorname{arctg}\left(\left(1+\frac{2 \mu_{1} a_{0}}{B_{0}\left(\frac{\mu_{1}^{2}}{8}+\mu_{2}\right)}\right) \frac{B_{0}\left(\frac{\mu_{1}^{2}}{8}+\mu_{2}\right)}{4 a_{0} \sqrt{2 \mu_{2}}}\right) \\
& -\frac{2 \mu_{1} a_{0} / B_{0}\left(\frac{\mu_{1}^{2}}{8}+\mu_{2}\right)}{\left.\frac{32 \mu_{2} a_{0}^{2}}{B_{0}^{2}\left(\frac{\mu_{1}^{2}}{8}+\mu_{2}\right)^{2}}+\frac{4 \mu_{1}^{2} a_{0}^{2}}{B_{0}^{2}\left(\frac{\mu_{1}^{2}}{8}+\mu_{2}\right)^{2}}-\frac{B_{0}\left(\frac{\mu_{1}^{2}}{8}+\mu_{2}\right)}{4 a_{0} \sqrt{2 \mu_{2}}} \operatorname{arctg} \frac{\mu_{1}}{2 \sqrt{2 \mu_{2}}}\right] .} .
\end{aligned}
$$

Certainly, $B_{0}\left(\frac{\mu_{1}^{2}}{8}+\mu_{2}\right)=16 a_{0}\left(1-\frac{\mu_{1}}{8} \pm \sqrt{1-1 / 4\left(\mu_{1}+\frac{\mu_{2}}{2}\right)}\right)$, and the denominator of the third term in (47) is $\frac{32 a_{0}^{2}\left(\frac{\mu_{1}^{2}}{8}+\mu_{2}\right)}{B_{0}\left(\frac{\mu_{1}^{2}}{8}+\mu_{2}\right)}=\frac{32 a_{0}^{2}}{B_{0}}$. This way we get

$$
\frac{\lambda_{2}}{\mu_{2}}=I_{2} \Rightarrow \lambda_{2}=\mu_{2} I_{2}=F_{2}\left(\mu_{1}, \mu_{2}\right)>0 .
$$

To simplify the previous formulas for $\lambda_{1}=F_{1}\left(\mu_{1}, \mu_{2}\right)$ and $\lambda_{2}=F_{2}\left(\mu_{1}, \mu_{2}\right)$ in $R$, we shall use the identity $\operatorname{arctg} \alpha-\operatorname{arctg} \beta=\operatorname{arctg} \frac{\alpha-\beta}{1+\alpha \beta}, \alpha>0, \beta>0$ and the series development $\operatorname{arctg} \alpha=\alpha-\frac{\alpha^{3}}{3}+\frac{\alpha^{5}}{5}+\cdots, \alpha$ near 0 . The smooth mapping $F=\left(F_{1}, F_{2}\right)$ maps $R$ onto some set $\tilde{R} \subset \mathbf{R}_{\lambda_{1}, \lambda_{2}}^{2}$. The solvability of (42) is reduced via formula (36) with $\left(\mu_{1}, \mu_{2}\right)$ written instead of $\left(\lambda_{1}, \lambda_{2}\right)$ to the invertibility of $R \stackrel{F}{\rightarrow} \tilde{R}$. For given $\left(\lambda_{1}, \lambda_{2}\right) \in \tilde{\mathbf{R}}$, we must find $\left(\mu_{1}, \mu_{2}\right) \in \mathbf{R}$. To find out $\left(\mu_{1}, \mu_{2}\right)$ into an explicit form is a difficult task. Below we write $L_{ \pm}=1-\frac{\mu_{1}}{8} \pm \sqrt{1-\frac{1}{4}\left(\mu_{1}+\frac{\mu_{2}}{2}\right)}$ in $\mathbf{R}$ and observe that $L_{+}>0, L_{-}=0 \Longleftrightarrow \mu_{1}=\mu_{2}=0$ and $L_{+} L_{-}=\frac{\mu_{2}}{8}+\frac{\mu_{1}^{2}}{64}>0$. Therefore, $L_{-}=\frac{\mu_{2} / 8+\mu_{1}^{2} / 64}{L_{+}}, 1 / 2 \leq L_{+} \leq 2$ in $\bar{R}, L_{+}(0,8)=1, L_{+}(0,0)=2$, $L_{+}(4,0)=\frac{1}{2}$.

Depending on the sign \pm in $L_{ \pm}$, we shall write $\lambda_{1 \pm}, \lambda_{2 \pm}$. Put $M_{ \pm}\left(\mu_{1}, \mu_{2}\right)=8 \mu_{2}+8 L_{ \pm} \mu_{1}+$ $\mu_{1}^{2}$. Standard but tiresome computations lead to the following formulas:

$$
\lambda_{1 \pm}=\frac{8 \pi \mu_{1}}{\sqrt{2 \mu_{2}}} \operatorname{arctg} \frac{16 L_{ \pm} \sqrt{2 \mu_{2}}}{M_{ \pm}},
$$




$$
\begin{aligned}
\lambda_{2 \pm}= & 16 \pi\left[\frac{1+\frac{\mu_{1}}{8 L_{ \pm}}}{\left(1+\frac{\mu_{1}}{8 L_{ \pm}}\right)^{2}+\frac{\mu_{2}}{8 L_{ \pm}^{2}}}-\frac{\frac{\mu_{1}}{8 L_{ \pm}}}{\frac{\mu_{2}}{8 L_{ \pm}^{2}}+\frac{\mu_{1}^{2}}{64 L_{ \pm}^{2}}}\right. \\
+ & \left.\frac{4 L_{ \pm}}{\sqrt{2 \mu_{2}}} \operatorname{arctg} \frac{16 L_{ \pm} \sqrt{2 \mu_{2}}}{M_{ \pm}}\right] .
\end{aligned}
$$

Certainly, $0<8 \mu_{2}+8 L_{-} \mu_{1}+\mu_{1}^{2}=8 \mu_{2}+\mu_{1} \frac{\frac{\mu_{2}}{8}+\frac{\mu_{1}^{2}}{64}}{L_{+}}+\mu_{1}^{2}$ for $\left(\mu_{1}, \mu_{2}\right) \neq 0$.

Evidently, $\lambda_{1 \pm}, \lambda_{2 \pm} \in C^{0}\left(\mu_{2}>0\right)$. Fix $\left(\mu_{1}^{0}, \mu_{2}^{0}\right) \neq 0$ and consider the cases $\lambda_{1 \pm}$. The function $M_{ \pm}$is positive near $\left(\mu_{1}^{0}, \mu_{2}^{0}\right)$ and therefore is bounded there, i.e., $\operatorname{arctg} L_{ \pm} \frac{16 \sqrt{2 \mu_{2}}}{M_{ \pm}}=$ $L_{ \pm} \frac{16 \sqrt{2 \mu_{2}}}{M_{ \pm}}+O\left(M_{ \pm}^{-2} \mu_{2}\right)$ near $\left(\mu_{1}^{0}, \mu_{2}^{0}\right)$. Thus, $\lim \lambda_{1 \pm}=0$ for $\mu_{1} \rightarrow \mu_{1}^{0}>0, \mu_{2} \rightarrow 0$.

This way we see that $\lambda_{1 \pm}, \lambda_{2 \pm} \in C^{0}(\bar{R} \backslash(0,0))$. Moreover, $\lambda_{2+}\left(\mu_{1}=0, \mu_{2}>0\right)=16 \pi \times$ $\left[\frac{1}{1+\frac{\mu_{2}}{8 L^{2}}}+\frac{4 L_{+}}{\sqrt{2 \mu_{2}}} \operatorname{arctg} \frac{2 \sqrt{2} L_{+}}{\sqrt{\mu_{2}}}\right], L_{+}\left(0, \mu_{2}\right)=1+\sqrt{1-\frac{\mu_{2}}{8}}, 0<\mu_{2}<8$. Thus, $\lim _{\mu_{2} \rightarrow 0} \lambda_{2+}\left(0, \mu_{2}\right)=$ $+\infty, \lambda_{2+}\left(\mu_{1}=0, \mu_{2}>0\right) \geq c_{0}>0$ for $0<c_{1} \leq \mu_{2}<8, \lambda_{2+}\left(\mu_{1}=0, \mu_{2}=8\right)=16 \pi\left(\frac{1}{2}+\frac{\pi}{4}\right)$, $\lambda_{1+}\left(\mu_{1}=4, \mu_{2}=0\right)=8 \pi, \lambda_{2+}\left(\mu_{1}=4, \mu_{2}=0\right)=+0$.

To study the behavior of $\lambda_{1 \pm}, \lambda_{2 \pm}$ near the origin, we define the paths $\mu_{1}=\mu_{2}^{\alpha}, \alpha>0$, $\mu_{2}>0$ leading to 0 in $\mathbf{R}_{\mu_{1}, \mu_{2}}^{2}$. Then

$$
\frac{1}{8 \pi \sqrt{2}} \lambda_{1+}\left(\mu_{2}^{\alpha}, \mu_{2}\right)=\mu_{2}^{\alpha-1 / 2} \operatorname{arctg} \frac{16 \sqrt{2} L_{+}}{8 \sqrt{\mu_{2}}+8 L_{+} \mu_{2}^{\alpha-1 / 2}+\mu_{2}^{2 \alpha}} .
$$

Standard computations give us that

$$
\lim _{\mu_{2} \rightarrow 0} \lambda_{1+}\left(\mu_{2}^{\alpha}, \mu_{2}\right)= \begin{cases}16 \pi, & 0<\alpha<1 / 2, \\ 8 \pi / \sqrt{2} \operatorname{arctg} 2 \sqrt{2}, & \alpha=1 / 2, \\ 0, & \alpha>1 / 2 .\end{cases}
$$

The evaluation of $\lim _{\mu_{2} \rightarrow 0} \lambda_{2+}\left(\mu_{2}^{\alpha}, \mu_{2}\right)$ is technically more complicated. In fact, according to (49), $\lambda_{2+}\left(\mu_{2}^{\alpha}, \mu_{2}\right)=I+I I+I I I$ and the third term contains $\operatorname{arctg} \frac{16 L_{+} \sqrt{2 \mu_{2}}}{M_{+}}$. We have to find $\lim _{\mu_{2} \rightarrow 0} I, \lim _{\mu_{2} \rightarrow 0} I I$ and $\lim _{\mu_{2} \rightarrow 0} I I I$.

We have to develop $I I\left(\mu_{2}^{\alpha}, \mu_{2}\right)$ and $I I I\left(\mu_{2}^{\alpha}, \mu_{2}\right)$ in Taylor series taking into account first several terms (not only one) in the corresponding finite sum. This way we come to the expression

$$
\lim _{\mu_{2} \rightarrow 0} \lambda_{2+}\left(\mu_{2}^{\alpha}, \mu_{2}\right)= \begin{cases}1, & 0<\alpha<1 / 3 \\ \frac{259}{3}, & \alpha=1 / 3 \\ +\infty, & 1 / 3<\alpha\end{cases}
$$

Geometrical visualization of $F$ is given on Fig. 1 .

Applying Sard's theorem to the smooth mapping $F$ in $R$, we conclude that, for almost each $\lambda=\left(\lambda_{1}, \lambda_{2}\right) \in \tilde{R}$, there exists such $\mu=\left(\mu_{1}, \mu_{2}\right) \in R$ that $\lambda=F(\mu)$ and $\frac{D\left(F_{1}, F_{2}\right)}{D\left(\mu_{1}, \mu_{2}\right)} \neq 0$. Therefore, the mapping $F$ is smoothly invertible near the point $\mu$ and $\mu=F^{-1}(\lambda)$. Putting $\mu=\mu(\lambda)$ into (36) with $B_{0}$ given by (41) we obtain the solution of (32). Certainly, in (36) and (41) the parameters $\mu_{1}, \mu_{2}$ are written instead of $\left(\lambda_{1}, \lambda_{2}\right)$. The case $\lambda_{1-}, \lambda_{2-}$ is left to the reader. This way Theorem 3 is proved. 


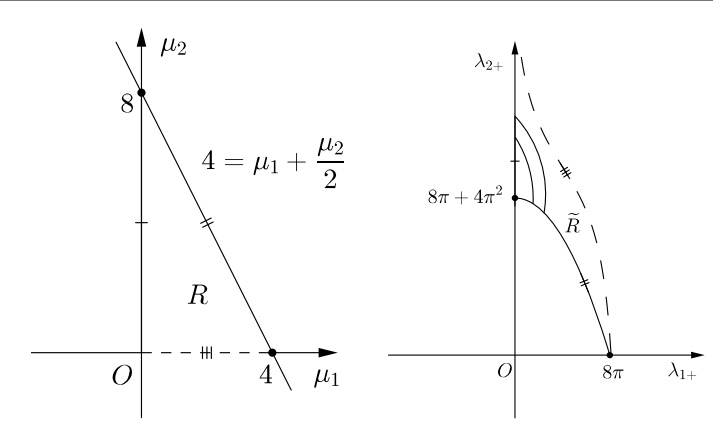

Figure 1 Graphs of $F$

Possible generalization of Theorem 3 concerns the stochastic Dirichlet problem for (42), namely

$$
\begin{aligned}
& \Delta u+\frac{\lambda_{1} e^{u / 2}+\lambda_{2}|x|^{2} e^{u}}{\int_{B_{1}}\left(\lambda_{1} e^{u / 2}+\lambda_{2}|x|^{2} e^{u}\right) d x}=0 \quad \text { in } B_{1}, \lambda_{1}>0, \lambda_{2}>0, \\
& \left.u\right|_{\partial B_{1}}=0 .
\end{aligned}
$$

Case (10) with $\frac{|x|^{2} e^{u}}{\int_{B_{1}}|x|^{2} e^{u} d x}$ was left to the reader.

\section{Proof of Theorem 4 and some applications}

At first we shall mention that the nonlocal BVP (14) is reduced to the local Cauchy problem

(13) after the standard change $\mu_{j}=\frac{\lambda_{j}}{\int_{B_{1}} e^{k_{j}^{u}} d x}, j=1, \ldots, n$.

We are looking for radially symmetric solution $u(r)$ of (13). After the polar change in

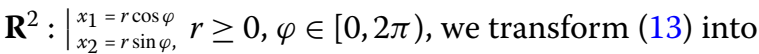

$$
r^{2} u_{r r}+r u_{r}+\sum_{j=1}^{n} \mu_{j} r^{\rho_{j}+2} e^{\kappa_{j} u}=0
$$

The Euler change $r=e^{t}, t=\log r, t \in(-\infty, \infty)$ enables us to obtain from (52) the Cauchy problem

$$
\begin{aligned}
& u_{t t}+\sum_{j=1}^{n} \mu_{j} e^{\left(\rho_{j}+2\right) t+\kappa_{j} u}=0 \quad \text { for } t \in(-\infty, 0], \\
& u(0)=u_{0} \\
& u_{t}(0)=\left.u_{r} r\right|_{r=1}=u_{1},
\end{aligned}
$$

as $\frac{\partial}{\partial r}=e^{-t} \frac{\partial}{\partial t}, \frac{\partial^{2}}{\partial r^{2}}=e^{-2 t}\left(\frac{\partial^{2}}{\partial t^{2}}-\frac{\partial}{\partial t}\right), u(r)=u\left(e^{t}\right) \equiv u(t)$.

Evidently, $\int_{B_{1}} e^{\kappa_{j} u} d x=2 \pi \int_{0}^{1} r e^{\kappa_{j} u(r)}=2 \pi \int_{-\infty}^{0} e^{\kappa_{j} u(t)+2 t} d t$. Therefore, $\mu_{j}=\frac{\lambda_{j}}{2 \pi \int_{-\infty}^{0} e^{\kappa_{j} u+2 t} d t}$, $1 \leq j \leq n$. 
Put $u=w(t)+A t$, where the constant $A$ is given by (i), Theorem 4 (a). Then (53) is rewritten as

$$
\begin{aligned}
& w_{t t}+\sum_{1}^{n} \mu_{j} e^{\left(\rho_{j}+2\right) t+\kappa_{j} w(t)+A \kappa_{j} t}=0, \quad t \leq 0, \\
& w(0)=u(0)=u_{0}, \\
& w^{\prime}(0)=u^{\prime}(0)-A=u_{1}-A .
\end{aligned}
$$

We shall write down $w(0)=w_{0}, w^{\prime}(0)=u_{1}-A=w_{1}$ for simplicity. Condition (i) of Theorem 4 (a) and the notation $B_{j}=\frac{\mu_{j}}{\lambda_{j}}>0$ imply: $e^{\kappa_{j} u(t)+2 t}=e^{\kappa_{j} w(t)-\rho_{j} t}, t \leq 0$. Therefore,

$$
\frac{d}{d t}\left(w^{\prime}\right)^{2}+2 \sum_{j=1}^{n} B_{j} \frac{d}{d t} e^{\kappa j w(t)}=0
$$

i.e.,

$$
\left(w^{\prime}\right)^{2}(t)+2 \sum_{j=1}^{n} B_{j} e^{\kappa_{j} w(t)}=2 C=\mathrm{const}
$$

where $2 C=w_{1}^{2}+2 \sum_{j=1}^{n} B_{j} e^{\kappa_{j} w_{0}}>0\left(C=C\left(w_{0}, w_{1}, \mu\right), \mu=\left(\mu_{1}, \ldots, \mu_{n}\right)\right)$. Consequently,

$$
w^{\prime}(t)= \pm \sqrt{2} \sqrt{C-\sum_{1}^{n} B_{j} e^{\kappa_{j} w(t)}}
$$

which implies that

$$
t= \pm \frac{1}{\sqrt{2}} \int_{w_{0}}^{w(t)} \frac{d z}{\sqrt{C-\sum_{1}^{n} B_{j} e^{\kappa_{j} z}}} .
$$

We shall consider in (56) the case with sign "+" in front of the integral. Thus, put

$$
F(y)=\frac{1}{\sqrt{2}} \int_{w_{0}}^{y} \frac{d z}{\sqrt{C-\sum_{1}^{n} B_{j} e^{\kappa_{j} z}}}, \quad \delta=F(0)<0 \text {, if } w_{0}>0 .
$$

Obviously, $F^{\prime}(y)>0, F\left(w_{0}\right)=0$. The function $g(z)=C-\sum_{1}^{n} B_{j} e^{\kappa_{j} z}$ has the following properties: $g(-\infty)=C, g(\infty)=-\infty$, and therefore $g(z)$ has a unique zero at some point $z_{0}$. Certainly, $w_{0}<z_{0}$ and $F\left(z_{0}\right)=l=\int_{w_{0}}^{z_{0}} \frac{d z}{\sqrt{2} \sqrt{g(z)}}>0$ as that integral is convergent for $y=z_{0}$ and divergent for $y=-\infty$. The mapping $F:\left(-\infty, z_{0}\right] \rightarrow(-\infty, l]$ is diffeomorphism. Moreover, $g(z) \sim C$ for $y \rightarrow-\infty$ implies that $t=F(y) \sim \frac{y}{\sqrt{2 C}}$ for $y \rightarrow-\infty$. On the other hand, if $z<z_{0}, z \approx z_{0}$, the following relation holds:

$$
\begin{aligned}
F(y)-F\left(z_{0}\right) & =\int_{z_{0}}^{y} \frac{d z}{\sqrt{2 g(z)}} \approx \int_{z_{0}}^{y}=\frac{d z}{\sqrt{2} \sqrt{\left(z_{0}-z\right)\left|g^{\prime}\left(z_{0}\right)\right|}} \\
& =-\frac{\sqrt{2}\left(z_{0}-y\right)^{1 / 2}}{\sqrt{\left|g^{\prime}\left(z_{0}\right)\right|}}, \quad y<z_{0}, F^{\prime}\left(z_{0}\right)=\infty, F\left(z_{0}\right)=l>0 .
\end{aligned}
$$




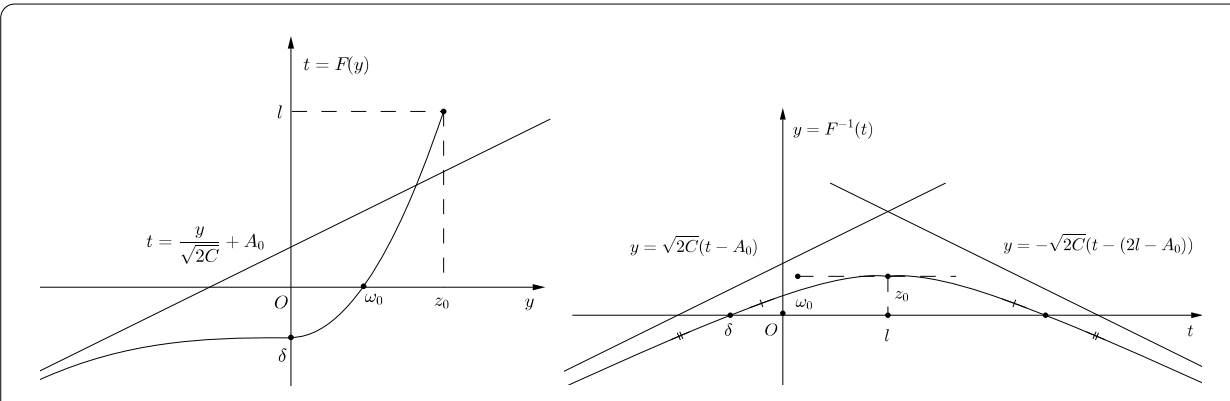

Figure 2 Graphs of $F$ and $F^{-1}$

The identity $t=F(y)$ implies that there exists a smooth inverse function $F^{-1}$ of $F$ such that $y=F^{-1}(t)$, i.e., $w(t)=F^{-1}(t), F^{-1}:(-\infty, l] \rightarrow\left(-\infty, z_{0}\right],\left(F^{-1}\right)^{\prime}(l)=0, F\left(w_{0}\right)=0 \Rightarrow w_{0}=$ $F^{-1}(0), y \sim \sqrt{2 C} t \Rightarrow y=F^{-1}(t) \sim \sqrt{2 C} t$ for $t \rightarrow-\infty$ and $y<z_{0}, y \sim z_{0} \Rightarrow F(y)-l \sim$ $-\sqrt{\frac{2}{\left|g^{\prime}\left(z_{0}\right)\right|}}\left(z_{0}-y\right)^{1 / 2}$. Thus, $y \sim z_{0}-\frac{\left|g^{\prime}\left(z_{0}\right)\right|}{2}(t-l)^{2}$ near $z_{0}$.

We can continue smoothly the function $F^{-1}(t)$ in an even way with respect to the point $l$, i.e., $F^{-1}(l+\tau)=F^{-1}(l-\tau)$ for each $\tau \geq 0$. Certainly, the continuation of $F^{-1}$ satisfies (55). A geometrical visualization of $F, F^{-1}$ is given in Fig. 2 .

This way we found out the solution $w=F^{-1}(t), t \in(-\infty, \infty), w(t)=u(t)-A t, w(0)=w_{0}$, $w^{\prime}(0)=w_{1}$. Then the solution of (53) we are looking for is $u\left(r, \mu, w_{0}, w_{1}\right)$ :

$$
\begin{aligned}
& u=w(t)+A t=F^{-1}(\log r)+A \log r, \quad A<0, w(r) \sim \sqrt{2 C} \log r, \quad r \rightarrow 0 ; \\
& w(r) \sim-\sqrt{2 C} \log r, \quad r \rightarrow \infty .
\end{aligned}
$$

In some cases the integral $F(y)$ can be rewritten in a more appropriate form after the change $e^{z}=\gamma$ :

$$
\frac{1}{\sqrt{2}} \int_{w_{0}}^{y} \frac{d z}{\sqrt{C-\sum_{1}^{n} B_{j} e^{\kappa_{j} z}}}=\frac{1}{\sqrt{2}} \int_{e^{w_{0}}}^{e^{y}} \frac{d \gamma}{\gamma \sqrt{C-\sum_{1}^{n} B_{j} \gamma^{\kappa_{j}}}} .
$$

If $\kappa_{j} \in \mathbf{N}$ or $\frac{\kappa_{j}}{\kappa_{1}} \in \mathbf{N}$, we have polynomial under the integral sign.

\section{Remark 2}

$$
\int_{B_{1}} e^{\kappa_{1} u} d x=2 \pi \int_{-\infty}^{0} e^{\kappa_{1} w+\kappa_{1} A t+2 t} d t=2 \pi \int_{-\infty}^{0} e^{\kappa_{1} w(t)-\rho_{1} t} d t<\infty
$$

if $\kappa_{1} \sqrt{2 C}>\rho_{1}$, as $w(t) \sim \sqrt{2 C} t, t \rightarrow-\infty . u$ is bounded at $r=0 \Longleftrightarrow \sqrt{2 C}=|A|$.

Example 4 Consider the case $\frac{\kappa_{1}}{\kappa_{2}}=2=\frac{\rho_{1}+2}{\rho_{2}+2}$. The simplest case is $\kappa_{2}=1 / 2, \kappa_{1}=1, \rho_{1}=2$, $\rho_{2}=0, A=-4$. Then the solution $u$ of (13) is given by the formula

$$
u=\log r^{-4}\left[\frac{4 C h\left(w_{0}\right) r^{\frac{1}{2} \sqrt{2 C}}}{\left(4 C B_{1}+B_{2}^{2}\right) r^{\sqrt{2 C}}+h^{2}\left(w_{0}\right)+2 B_{2} h\left(w_{0}\right) r^{\frac{\sqrt{2 C}}{2}}}\right]^{2},
$$


where $h\left(w_{0}\right)=2 \sqrt{C} \sqrt{C e^{-w_{0}}-B_{1}-B_{2} e^{-\frac{1}{2} w_{0}}}+2 C e^{-\frac{1}{2} w_{0}}-B_{2} ; B_{1}, B_{2}$ are the coefficients of the quadratic polynomial participating in

$$
F(y)=\frac{1}{\sqrt{2}} \int_{w_{0}}^{y} \frac{d z}{\sqrt{C-B_{1} e^{z}-B_{2} e^{z / 2}}},
$$

$2 C=w_{1}^{2}+B_{1} e^{w_{0}}+B_{2} e^{\frac{w_{0}}{2}}$. The change $p=e^{\frac{z}{2}}$ reduces the computation of $F(y)$ to the computation of $\int \frac{d p}{p \sqrt{C-B_{1} p^{2}-B_{2} p}}($ see $[8], 380.11,[5])$.

The proof of Theorem 4 is completed.

\section{Acknowledgements}

This research is financially supported by the project KP-06-H28/7.

\section{Funding}

There are no sources of funding for the research reported in this article.

\section{Availability of data and materials}

All data generated or analysed during this study are included in this published article (and its supplementary information files).

\section{Competing interests}

The authors declare that they have no competing interests.

\section{Authors' contributions}

PP formulated the main theorems. AS was involved in the proofs as well as in geometrical interpretations shown on the figures. All authors read and approved the final manuscript.

\section{Publisher's Note}

Springer Nature remains neutral with regard to jurisdictional claims in published maps and institutional affiliations.

Received: 24 June 2021 Accepted: 6 August 2021 Published online: 19 August 2021

\section{References}

1. Arnold, V.: Ordinary Differential Equations. Springer, Berlin (1992)

2. Bartolucci, D., Leoni, F., Orsina, L., Ponce, A.: Semilinear equations with exponential nonlinearity and measure data. Ann. Inst. Henri Poincaré, Anal. Non Linéaire 22(6), 799-815 (2005)

3. Bartolucci, D., Lin, C.: Existence and uniqueness for mean field equations on multiply connected domains at the critical parameter. Math. Ann. 359(1-2), 1-44 (2014)

4. Brezis, H., Merle, F.: Uniform estimates and blow-up behaviour for solutions of $-\Delta v=v(x) e^{u}$ in two dimensions. Commun. Partial Differ. Equ. 16(8,9), 1223-1253 (1991)

5. Byrd, P., Friedman, M.: Handbook of Elliptic Integrals for Engineers and Scientists. Springer, NY (1971)

6. Chavanis, B.: Statistical mechanics of the two dimensional vortices and stellar systems, dynamics and thermodynamics of systems with long range interactions. In: Les Houches, Lecture Notes in Phys., vol. 602, pp. 208-289. Springer, Berlin (2002)

7. De Marchis, F., Ricciardi, T.: Existence of stationary turbulent flows with variable positive vortex intensity. Nonlinear Anal., Real World Appl. 38, 222-244 (2017)

8. Dwight, H.: Tables of Integrals and Other Mathematical Data. Macmillan Co., NY (1961)

9. Eyink, G., Sreenivasan, K.: Onsager and the theory of hydrodynamic turbulnece. Rev. Mod. Phys. 78(1), 87-135 (2006)

10. Gidas, B., Ni, W.M., Nirenberg, L.: Symmetry and related properties via the maximum principle. Commun. Math. Phys, 68(3), 209-243 (1979)

11. Hoffman, K.: Banach Spaces of Analytic Functions. Prentice-Hall, Englewood Cliffs (1962)

12. Jevnikar, A., Yang, W.: Analytic aspects of Tzitzeika equation: blow up analysis and existence results. Calc. Var. Partial Differ. Equ. 56(2), Article ID 43 (2017)

13. Malchiodi, A.: Topological methods for an elliptic equation with exponential nonlinearities. Discrete Contin. Dyn. Syst. 21(1), 277-294 (2008)

14. Moser, J.: A sharp form of an inequality by N. Trudinger. Indiana Univ. Math. J. 20, 1077-1092 (1970/71)

15. Onsager, L.: Statistical hydrodynamics. Nuovo Cimento (9) 6(2)-Supplemento, 279-287 (1949)

16. Pistoia, A., Ricciardi, R.: Concentrating solutions for a Liouville type equation with variable intensities in 2-D turbulence. Nonlinearity 29(2), 271-297 (2016)

17. Poliakovsky, A., Tarantello, G.: On a planar Liouville type problem in the study of selfgravitating strings. J. Differ. Equ. 252(5), 1327-1348 (2012)

18. Popivanov, P.: Explicit formulas to the solutions of Dirichlet problem for equations arising in geometry and physics. C. R. Acad. Bulgare Sci. 68(1), 19-24 (2015) 
19. Popivanov, P., Nikolov, N.: On the Dirichlet problem to the Liouville equation. Explicit formulas. C. R. Acad. Bulgare Sci. 69(11), 1389-1400 (2016)

20. Popivanov, P., Slavova, A.: Nonlinear Waves. An Introduction. Series on Analysis, Appl. and Comp., vol. 4. World Scientific, Singapore (2011)

21. Popivanov, P., Slavova, A.: Full classification of the travelling wave solutions of Fornberg-Whitham equation. Solutions into explicit form. C. R. Acad. Bulgare Sci. 65(5), 563-574 (2012)

22. Popivanov, P., Slavova, A.: Nonlinear Waves. A Geometrical Approach. Series on Analysis, Appl. and Comp., vol. 9. World Scientific, Singapore (2019)

23. Popivanov, P., Slavova, A., Zecca, P.: Regularizing property of the solutions of a dissipative semilinear wave equation. C. R. Acad. Bulgare Sci. 63(7), 861-870 (2010)

24. Prajapat, J., Tarantello, G.: On a class of elliptic problems in $\mathbf{R}^{2}$ :symmetry and uniqueness results. Proc. R. Soc. Edinb. 131A, 967-985 (2001)

25. Ricciardi, T., Takahashi, R.: On radial two-species Onsager vortices near the critical temperature. Indiana Univ. Math. J. 68(6), 1903-1926 (2019)

26. Suzuki, T.: Global analysis for a 2-dimensional elliptic eigenvalue problem with the exponential nonlinearity. Ann. Inst. Henri Poincaré, Anal. Non Linéaire 9(4), 367-397 (1992)

27. Suzuki, T.: Mean Field Theories and Dual Variations: Mathematical Structures of the Mesoscopic Model, 2nd edn. Atlantis Studies in Math. for Engineering and Science., vol. 11. Atlantis Press, Paris (2015)

28. Tribuzy De Azevedo, R., Guadalupe, I.: Minimal immersions of surfaces into 4-dimensional space form. Rend. Semin. Mat. Univ. Padova 73, 1-13 (1985)

29. Yang, Y: Self-duality of the gauge filed equations and the cosmological constant. Commun. Math. Phys. 162(3), 481-498 (1994)

30. Zhou, C.: Existence result for meanfield equation on the equilibrium turbulence in the super critical case. Commun. Contemp. Math. 13(4), 659-673 (2011)

31. Zygmund, A.: Trigonometric Series. Vol. 1 and 2, 2nd edn. Cambridge University Press, London (1959)

\section{Submit your manuscript to a SpringerOpen ${ }^{\circ}$ journal and benefit from:}

- Convenient online submission

- Rigorous peer review

- Open access: articles freely available online

- High visibility within the field

- Retaining the copyright to your article

Submit your next manuscript at $\boldsymbol{\nabla}$ springeropen.com 